\title{
Initial allocation of carbon emission permits in power systems
}

\author{
Tan WANG ${ }^{1}$, Xu WANG ${ }^{1}$, Yu GONG ${ }^{1}$, Chuanwen JIANG ${ }^{1}$, \\ Fengjia XIONG ${ }^{2}$, Lei $\mathrm{LI}^{3}$, Yan $\mathrm{ZHAO}^{3}$
}

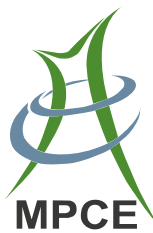

\begin{abstract}
Initial allocation modes and schemes are studied in this paper to provide guidelines for allocation of carbon emission permits in power system. We first introduce different allocation modes and the need to apply free allocation to assignability of emission permits. Then, we compare two different allocation schemes, which are based on historical emissions and generation performance standard. Further, a new allocation scheme based on Boltzmann distribution is proposed. Finally, a case study on
\end{abstract}

CrossCheck date: 18 November 2015

Received: 21 September 2014/Accepted: 21 December 2014/ Published online: 18 February 2016

(C) The Author(s) 2016. This article is published with open access at Springerlink.com

$\triangle$ Tan WANG

wt_sjtu@163.com

Xu WANG

wangx198912@163.com

Yu GONG

gongyu200810@126.com

Chuanwen JIANG

jiangcw@sjtu.edu.cn

Fengjia XIONG

847893398@qq.com

Lei LI

lilei@smepc.com

Yan ZHAO

zhaoyan@smepc.com

1 Electrical Engineering Department, Shanghai Jiao Tong University, Shanghai 200240, China

2 Henry Samueli School of Engineering and Applied Science, University of California, Los Angeles 90024, USA

3 Electric Power Transaction Center, Shanghai Municipal Electric Power Company, Shanghai 200122, China
Shanghai power grid in China is conducted to compare the allocation effects of these schemes respectively. The analytical results show that Boltzmann distribution based scheme has the best performance and should be adopted when developing initial allocation of carbon emission permits in Chinese power systems.

Keywords Carbon emission permits, Initial allocation, Generation performance standard, Boltzmann distribution

\section{Introduction}

The climate change resulted by global greenhouse gas emission has become a significant issue that drew attention from the international community. Therefore, controlling the emission of greenhouse gas, mainly the $\mathrm{CO}_{2}$, becomes a critical way to alleviate the effect of global warming on human activities. In the past decades, several policy instruments have been developed to attempt to mitigate climate change and reduce carbon emissions. Carbon emission trading originated from the West, and it has developed into an effective measure to reduce carbon emissions worldwide.

Chinese government takes active part in energy saving and emission reduction. On the United Nations Climate Change conference in 2009, Copenhagen, Chinese government proposed that carbon dioxide emissions per unit of GDP would be reduced by $40 \% \sim 45 \%$ before 2020 . In order to reach the announced reduction target, seven regions in China has launched pilot carbon trading markets of their own since 2013.

To encourage carbon trading, a fundamental problem needed to be settled is how to set the initial allocation approaches, which is also what this paper focuses on. The reasonability of allocation mechanisms determines whether 
emission trading system could work efficiently [1]. If the mechanisms are not reasonable, imbalance between supply and demand may deteriorate, causing chaos in the carbon trading market. There might even be some vicious arbitrages as a result.

Study on initial allocation mainly considers the mode and scheme of allocation. The mode of allocation refers to how emission permits are allocated, whereas the scheme concerns about indexes of allocation. Much previous literature analyzed allocation of carbon emission permits from these two aspects. Reference [2] presented the impact of emission allocation criteria on the emission reduction and operational cost of the power system. Reference [3] compared four methods by models, which are based on historical emissions levels, updated emissions levels, updated production levels and historical production levels. In [4-7], allocation methods based on power generation and fuel type were discussed. A two-level allocation mechanism based on regional comparison which can allocate the carbon emission permits rationally and fair was proposed in [8]. Reference [9] presented an optimal economic efficiency model of emission trading, analyzing the economic efficiency of emission trading with different proportion of auction in initial allocation. It was proposed in [10] that the method based on emissions performance standard can allocate emission permits effectively. Reference [11] examined bidders' bidding behavior and carbon emission rights allocations under the English auction with "going, going, gone" ending rule and the sequential ascending auction, respectively. In [12], a multi-stage profit model was developed to analyze the ETS-covered enterprises' product prices and emission reduction behaviors under different allocation rules. Benchmark, grandfathering and the Shapley value were employed in [13] to simulate the initial allocation of carbon emission allowances of the three power plants in Pudong New District, Shanghai, China.

To design allocation schemes, factors we should take into account are effectiveness, fairness, and feasibility [14]. There are two types of prevalent schemes: grandfathering and benchmarking $[15,16]$. And specific schemes in previous researches are as follows.

1) Power generation based allocation

2) Generation performance standard (GPS) based allocation

3) Fuel type based allocation

4) Installed capacity based allocation

5) Benchmark historical emissions based allocation

6) Synthetic allocation

7) Exergy based allocation method for carbon dioxide emissions from cogeneration [17]

However, these schemes cannot meet the expectation of effectiveness, and there still remains a few problems. For instance, considering historical emissions based allocation, the selection of base year is controversial and the historical data may be arduous to obtain, especially for newly installed power plants.

In this paper, an allocation scheme based on Boltzmann distribution is presented. In terms of indexes, it is developed from GPS based scheme, taking into account both power generation and GPS. With the application of Boltzmann distribution and entropy maximization theorem, it is also a neoteric scheme.

The rest of this paper unfolds as follows: allocation modes are discussed in Section 2. Then, we give a brief introduction to two typical allocation schemes in Section 3. Section 4 presents a new scheme based on Boltzmann distribution. Section 5 provides a case study based on Shanghai power grid to compare the allocation effects of three allocation schemes. Conclusions are drawn in Section 6.

\section{Allocation modes}

There are three kinds of initial allocation modes: free allocation, fixed-price and auction [18]. Table 1 shows the modes of several main emission trading markets.

In free allocation mode, power plants get emission permits for free, meeting their needs of electrical production. Some emission permits beyond their demand may be obtained, inducing extra profit opportunities. The advantage is that it provides power plants with asset which can be traded in the market without increasing their production cost. But it may weaken their motivation of emission reduction.

In auction or fixed price mode, power producers must pay money for the emission permits. It is beneficial to the internalization of carbon emission costs [19], motivating power plants to reduce emission. However, the disadvantage is that it will increase their production costs, affecting their market competitiveness or even their regular production.

Chinese carbon trading market is not mature yet, and the scope of implementation is quite limited. For the sake of reducing resistance of policy implementation, free allocation is the mode we should apply to allocate emission permits.

\section{Allocation schemes}

In this section, two typical allocation schemes are briefly introduced: historical emissions based allocation and GPS based allocation. 
Table 1 Modes of main emission trading markets

\begin{tabular}{|c|c|}
\hline Markets & Allocation modes \\
\hline European Union & $\begin{array}{l}\text { In the first and second stage, free allocation is the mainstream, and a small quantity of auction is used as } \\
\text { supplement. In the third stage, only auction is applied. }\end{array}$ \\
\hline Australia & $\begin{array}{l}\text { In the first stage, free allocation and fixed-price are used. In the second stage, free allocation and auction are } \\
\text { applied. }\end{array}$ \\
\hline New Zealand & Free allocation and fixed-price \\
\hline America & Free allocation and auction \\
\hline
\end{tabular}

\subsection{Historical emissions based allocation}

It is the most commonly used scheme and is often called "grandfathering". Many countries in European Union like Netherlands, France and Czech Republic all employ this scheme. Permits allocated to an emission unit are proportional to the proportion of its carbon emission in the total emissions by all units in base year, as shown by:

$P_{i}=\frac{E_{i_{\text {base }}}}{E_{\text {total base }}} P_{\text {total }}$

where $P$ is permits; and $E$ is carbon emission.

The selection of base year is of great importance to this scheme, and permits allocated will be distinct with different base years.

\subsection{GPS based allocation}

GPS reflects the emission intensity of power plants and it is defined as the emissions per unit of electricity production [20], as shown by:

$S_{\text {target }}=\frac{\bar{E}}{\bar{G}}$

where $S$ is generation performance standard; $\bar{E}$ the permitted carbon emissions in the target year; and $\bar{G}$ the total power generation of target year.

With known power generation of a power plant $G_{i}$, permits allocated are as:

$P_{i}=S_{\text {target }} G_{i}$

\subsection{A comparison}

Historical emissions based allocation is prevalent. It is simple and only a small amount of data is needed. The existing problems are that the selection of base year is controversial and historical data may be arduous to obtain, especially for newly installed power plants. What's more, the future permits that power plants will acquire are proportional to their historical emissions, which will kill the motivation to reduce emission.
GPS based allocation is an impartial and effective scheme. The data needed is easy to obtain. Compared with historical emissions based allocation, it is more practical for China, whose electricity consumption is continuously increasing [21].

By this comparison, we can conclude that GPS based scheme is more appropriate for the application of Chinese power systems.

\section{Boltzmann distribution based allocation}

\subsection{Introduction}

Boltzmann distribution is a kind of probability distribution, and it has been widely employed in the fields of physics and chemistry, most commonly in statistic mechanics. In physics, Boltzmann distribution yields the equilibrium probability distribution of a physical system in its energy sub states. The probability that a particle can be found in the substate is inversely proportional to the exponential function of the substate energy $E_{i}$, as shown by:

$p_{i} \propto e^{-\beta E_{i}}$

Table 2 Basic information of participated plants

\begin{tabular}{lll}
\hline Power plant & $\begin{array}{l}\text { Installed } \\
\text { capacity (MW) }\end{array}$ & $\begin{array}{l}\text { Emission } \\
\text { intensity }(\mathrm{g} / \mathrm{kWh})\end{array}$ \\
\hline 1 & 2000 & 744 \\
2 & 1320 & 768.6 \\
3 & 2000 & 744 \\
4 & 600 & 821.8 \\
5 & 600 & 821.8 \\
6 & 1200 & 815.1 \\
7 & 1200 & 821.8 \\
8 & 1200 & 795.4 \\
9 & 1200 & 795.4 \\
10 & 1800 & 795.1
\end{tabular}


Boltzmann distribution satisfies entropy maximization theorem. The concept of entropy was proposed by German physicist Rudolf Clausius to describe the uniformity of space energy distribution. American electrical engineer Shannon introduced the concept of information entropy, using it to measure the unpredictability of an event. E.T. Jaynes proposed entropy maximization theorem in 1957. According to the theorem, in making inferences on the basis of partial information, we must use the probability distribution which has maximum entropy subject to whatever is known. And he obtained expression of the probability as (5) [22]:

$p_{i}=e^{-\lambda-\mu f\left(x_{i}\right)}$

Jaynes concluded that the theory of maximum entropy inference was identical in mathematical form with the rules of calculation provided by statistical mechanics. The Hungarian mathematician Csiszar, winner of Shannon prize, had also proved that for a group of information which is not contradictory, there exist unique entropy maximization models and their mathematical forms are exponential functions.

At present, a series of physical conceptions like entropy maximization have been applied to economic issues and have achieved a great success. Some economists and physicists have introduced entropy concepts into the field of economics and have discussed the distribution of economic systems and their evolution. Besides, econophysicists have employed a stochastic process in describing the dynamics of individual wealth or income and in deriving their probability distributions. Banerjee and Yakovenko showed in one of their published papers in 2010 that the common theme of the distribution of money, income, and global energy consumption is entropy maximization for the partitioning of a limited resource among multiple agents [23].

Ji-Won Park of Cornell University brought entropy maximization to international emissions trading via the Boltzmann distribution, providing guidelines for allocating emissions permits among multiple countries. In his paper, the concept of physical particle is replaced by unit emissions permit. The concept of the physical substates is replaced by individuals of the participating countries. The probability that emission permits are allocated to a country $i$ is as (6) [24]:

$p_{i} \propto C_{i} e^{-\beta E_{i}}$

where $C_{i}$ is total population of a country; and $E_{i}$ is the negative value of $\mathrm{CO}_{2}$ emissions per capita of the country.

Emission permits will be allocated according to $p_{i}$. Considering the total number of available unit emission permits $(N)$, the allocated permits of country $i$ is as:
$N \frac{C_{i} e^{-\beta E_{i}}}{\sum_{i=1}^{n} C_{i} e^{-\beta E_{i}}}$

Boltzmann distribution provides the most probable distribution of a physical system at equilibrium. When brought to initial permits allocation, it provides the most probable (or fair) allocation among multiple countries.

And it is pointed out by Ji-Won Park that Boltzmann distribution is a simple yet versatile, flexible method, and it can be applied not only to permits allocation in emissions trading but also to other economic and environmental problems.

Besides, there is another paper introduces Boltzmann distribution to allocation of emission permits among enterprises, and a distribution mechanism based on Boltzmann distribution combined with Gail-Shapley game is proposed [25].

On the basis of these two papers, we can see that it is feasible to apply Boltzmann distribution to the allocation of emission permits.

The paper of Ji-Won Park mainly discusses allocation of permits among multiple countries. The other focuses on allocation among enterprises, and Gail-Shapley game is utilized in its mechanism. However, when we consider allocation in power system, things will be different. Up to now, there are no papers researching the application of Boltzmann distribution to emission permits allocation in power systems. In the following section, an allocation scheme based on Boltzmann distribution which is different from the two above-mentioned schemes both in the math expressions and indexes is presented.

\subsection{Allocation model}

If we regard the initial allocation of emission permits as an uncertain problem, according to entropy maximization theorem, its probability distribution is the one which has maximum entropy and the mathematical forms are exponential functions. Here, we choose Boltzmann distribution as the probability distribution. Then, the probability that emission permits are allocated to power plant $i$ as:

$p_{i} \propto G_{i} e^{-\beta S_{i}}$

where $G_{i}$ is annual power generation; and $S_{i}$ is the negative value of GPS.

The constraint of total available permits is formulated as:

$\sum_{i=1}^{n} P_{i}=N$

Permits are allocated based on $p_{i}$, with the constraint of total available permits, as shown by: 
Table 3 Power generation of participated plants

\begin{tabular}{lccc}
\hline Power plant & Power generation of $2011(\mathrm{GW})$ & Power generation of $2012(\mathrm{GW})$ & Power generation of 2013 $(\mathrm{GW})$ \\
\hline 1 & 12456.87652 & 11952.96057 & 11504.42308 \\
2 & 6862.217806 & 7738.615158 & 7603.199339 \\
3 & 12600.69149 & 11917.52615 & 11504.88604 \\
4 & 3791.926044 & 3743.462062 & 3558.326201 \\
5 & 2968.882571 & 2218.247300 & 2216.695645 \\
6 & 6207.236688 & 5006.928253 & 5229.146510 \\
7 & 7680.610299 & 7710.012842 & 7874.626166 \\
8 & 7411.846747 & 6472.787289 & 6708.147760 \\
9 & 6424.783981 & 5488.567815 & 5410.160742 \\
10 & 10561.44464 & 9690.080223 & 9823.769673 \\
\hline
\end{tabular}

Table 4 Emissions of participated power plants

\begin{tabular}{llll}
\hline Power plants & Carbon emissions of $2011\left(10^{4} \mathrm{t}\right)$ & Carbon emissions of $2012\left(10^{4} \mathrm{t}\right)$ & Carbon emissions of $2013\left(10^{4} \mathrm{t}\right)$ \\
\hline 1 & 926.7916129 & 889.3002665 & 855.9290772 \\
2 & 527.4300606 & 594.7899610 & 584.3819012 \\
3 & 937.4914466 & 886.6639456 & 855.9635214 \\
4 & 311.6204823 & 307.6377123 & 292.4232472 \\
5 & 243.9827697 & 182.2955631 & 182.1680481 \\
6 & 505.9518624 & 408.1147219 & 426.2277320 \\
7 & 631.1925544 & 633.6088554 & 647.1367783 \\
8 & 589.5382903 & 514.8455010 & 533.5660728 \\
9 & 511.0273178 & 436.5606840 & 430.3241854 \\
10 & 839.7404636 & 770.4582785 & 781.0879267 \\
\hline
\end{tabular}

$$
P_{i}=\frac{N \cdot G_{i} e^{-\beta S_{i}}}{\sum_{i=1}^{n} G_{i} e^{-\beta S_{i}}}
$$

The value of $\beta$ is of much importance, and it will directly affect the allocation results. It should be set to the value which can minimize the value of $y$, whose expression is:

$\min y=\sum_{i=1}^{n}\left(P_{i}-D_{i}\right)^{2}$

where $P_{i}$ is the allocated permits; and $D_{i}$ is the demand of carbon emission.

We can get the value of $\beta$ by fitting historical data of two years.

In this scheme, GPS represents environmental factors, and annual power generation stands for economic aspects. Thus, the allocation scheme based on Boltzmann distribution reflects a tradeoff between environment and economy.

What's more, if we put aside Boltzmann distribution theory, only focusing on its expression, it can be seen that

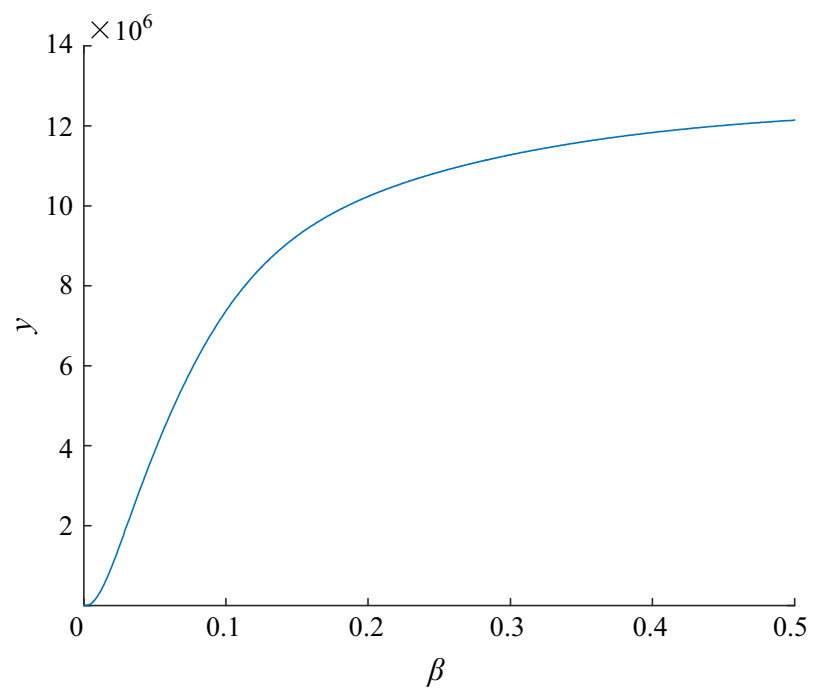

Fig. $1 y, \beta$ curve $(0<\beta<0.5)$

the emission permits allocated to power plants are proportional to the power generation and GPS. GPS based allocation and power generation based allocation have been 


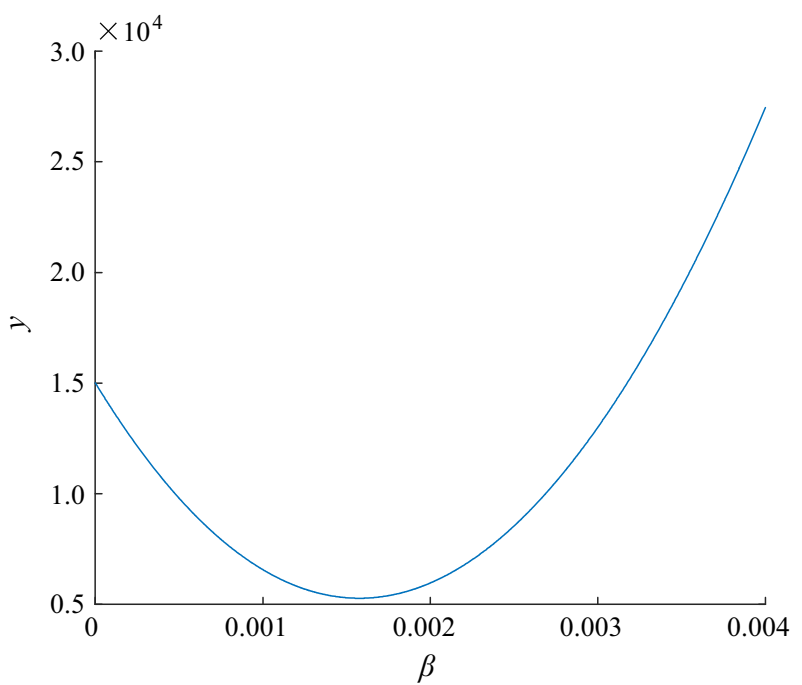

Fig. $2 y, \beta$ curve $(0<\beta<0.004)$ proved by various literatures to be feasible. With a combination of these two schemes, the results of our allocation scheme should be better.

\section{Case study}

In this section, a case study based on Shanghai power grid is presented to compare the allocation effects of GPS based scheme, historical emissions based scheme, and Boltzmann distribution based scheme.

Data from ten power plants of Shanghai power grid is utilized in this case study. Power generation and other basic information are listed in Table 2 and Table 3.

According to power generation and emission intensity listed above, we can get the emissions of participated power plants as shown in Table 4.

Table 5 Allocation results of Boltzmann distribution based scheme

\begin{tabular}{llllc}
\hline Power plants & Emissions of $2013\left(10^{4} \mathrm{t}\right)$ & $P_{i}$ & Allocated permits & Difference \\
\hline 1 & 855.9290772 & 0.1514 & 842.9197 & 13.0093772 \\
2 & 584.3819012 & 0.1040 & 579.1503 & 5.23160120 \\
3 & 855.9635214 & 0.1514 & 842.9914 & 12.9721214 \\
4 & 292.4232472 & 0.0529 & 294.8217 & -2.3984528 \\
5 & 182.1680481 & 0.0330 & 183.6606 & -1.4925519 \\
6 & 426.2277320 & 0.0770 & 428.7029 & -2.4751680 \\
7 & 647.1367783 & 0.1172 & 652.4674 & -5.3306217 \\
8 & 533.5660728 & 0.0957 & 533.1149 & 0.4511728 \\
9 & 430.3241854 & 0.0772 & 429.9202 & 0.4039854 \\
10 & 781.0879267 & 0.1401 & 780.2837 & 0.8042267
\end{tabular}

Table 6 Allocation results of historical emissions based scheme

\begin{tabular}{llrr}
\hline Power plants & Emissions of $2013\left(10^{4} \mathrm{t}\right)$ & Allocated permits & Difference \\
\hline 1 & 855.9290772 & 856.5321 & -0.6030228 \\
2 & 584.3819012 & 487.4459 & 96.9360012 \\
3 & 855.9635214 & 866.4208 & -10.4572786 \\
4 & 292.4232472 & 287.9967 & 4.42654720 \\
5 & 182.1680481 & 225.4866 & -43.3185519 \\
6 & 426.2277320 & 467.5959 & -41.3681680 \\
7 & 647.1367783 & 583.3422 & 63.79457830 \\
8 & 533.5660728 & 544.8457 & -11.2796272 \\
9 & 430.3241854 & 472.2866 & -41.9624146 \\
10 & 781.0879267 & 776.0802 & 5.00772670 \\
\hline
\end{tabular}


Table 7 Allocation results of GPS based scheme

\begin{tabular}{llrr}
\hline Power plants & Emissions of $2013\left(10^{4} \mathrm{t}\right)$ & Allocated permits & Difference \\
\hline 1 & 855.9290772 & 896.7242 & -40.7951228 \\
2 & 584.3819012 & 592.6302 & -8.2482988 \\
3 & 855.9635214 & 896.8005 & -40.8369786 \\
4 & 292.4232472 & 277.3619 & 15.0613472 \\
5 & 182.1680481 & 172.7840 & 9.3840481 \\
6 & 426.2277320 & 407.6067 & 18.621032 \\
7 & 647.1367783 & 613.8274 & 33.3093783 \\
8 & 533.5660728 & 522.9060 & 10.6600728 \\
9 & 430.3241854 & 421.6873 & 8.6368854 \\
10 & 781.0879267 & 765.7044 & 15.3835267 \\
\hline
\end{tabular}

Table 8 Difference rates of the three schemes

\begin{tabular}{lccr}
\hline Power plants & Boltzmann distribution $(\%)$ & Historical emissions $(\%)$ & GPS $(\%)$ \\
\hline 1 & 1.52 & -0.07 & -4.77 \\
2 & 0.90 & 16.59 & -1.41 \\
3 & 1.52 & -1.22 & -4.77 \\
4 & -0.82 & 1.51 & 5.15 \\
5 & -0.82 & -23.78 & 5.15 \\
6 & -0.58 & -9.71 & 4.37 \\
7 & -0.82 & 9.86 & 5.15 \\
8 & 0.08 & -2.11 & 2.00 \\
9 & 0.09 & -9.75 & 2.01 \\
10 & 0.10 & 0.64 & 1.97 \\
\hline
\end{tabular}

To allocate permits using Boltzmann distribution based scheme, we need to get $\beta$ according to (11). Total available permits are carbon emissions of 2012 reduced by $1 \%$, i.e., 55680327 tons. $G_{i}$ is the power generation of 2013, and $D_{i}$ is the carbon emissions of 2013.

First, $y, \beta$ curve is plotted in Fig. 1 and Fig. 2.

It can be seen from Fig. 1 and Fig. 2 that the minimum value of $y$ is reached when $\beta$ takes a value between 0.001 and 0.002. After zooming in the figure, $\beta$ is set to 0.00158 .

Then, permits are allocated according to (10), and results are shown in Table 5.

As for historical emissions based scheme, base year is 2011, and the total available permits are also 55680327 tons. The allocation results are shown in Table 6.

Target year of GPS based allocation is 2013, and the total available permits are still 55680327 tons. The results are shown in Table 7.

Based on Table 5, Table 6 and Table 7, difference rates of allocated permits and the demand can be calculated, as shown in (12):
$r=\frac{D_{i}-P_{i}}{L}$

The results are shown in Table 8 and Fig. 3 .

According to Table 8 and Fig. 3, it is obvious that when carbon emission permits are allocated via Boltzmann distribution based scheme, the distribution of difference rate is more even and smooth, with a range from $-0.9 \%$ to $1.6 \%$. When permits are allocated using other two schemes, difference rates are far beyond this range. So that Boltzmann distribution based allocation is a fair scheme which can provide approving permits for most power plants on the premise of satisfying constraint of total available permits.

\section{Conclusion}

This paper mainly discusses the initial allocation modes and schemes, aiming to provide guidelines for allocation of carbon emission permits in power system. Regarding allocation modes, it is concluded that we should apply free allocation to allocate emission permits. 


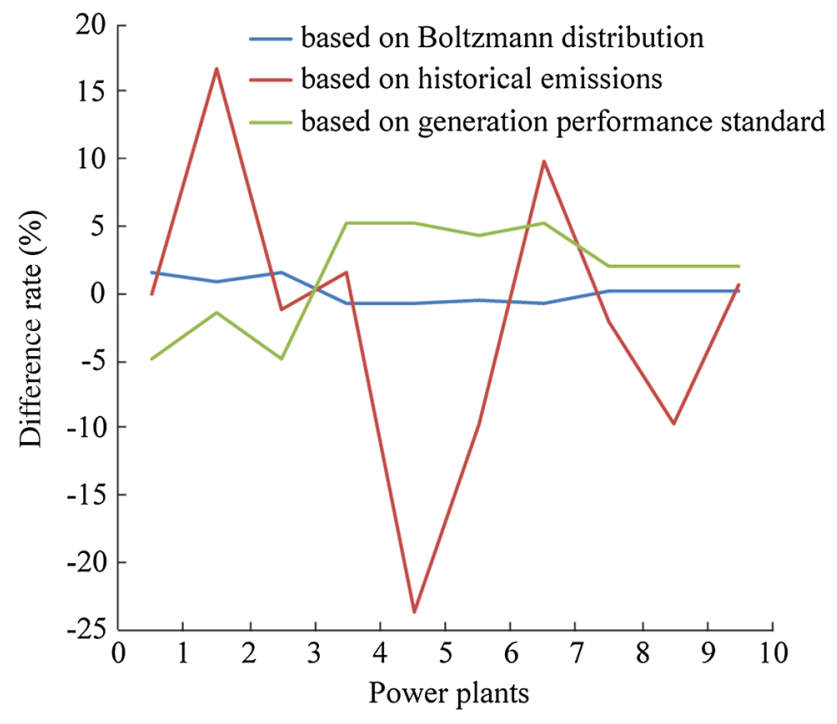

Fig. 3 Comparison of the three allocation schemes

As for schemes, the discussion of section 3.3 suggests that compared with historical emissions based allocation, GPS based allocation is more appropriate for the implementation in China. Boltzmann distribution based scheme is developed from GPS based scheme in terms of indexes. Results of the case study indicate that Boltzmann distribution based scheme performs the best among the three allocation schemes. Accordingly, we should employ Boltzmann distribution based scheme when developing initial allocation of carbon emission permits in Chinese power system.

Acknowledgements This work was supported by State Grid Corporation of China (No. 520900140069).

Open Access This article is distributed under the terms of the Creative Commons Attribution 4.0 International License (http:// creativecommons.org/licenses/by/4.0/), which permits unrestricted use, distribution, and reproduction in any medium, provided you give appropriate credit to the original author(s) and the source, provide a link to the Creative Commons license, and indicate if changes were made.

\section{References}

[1] Li XI, Yu CW (2010) Impacts of emission trading on carbon, electricity and renewable markets: a review. In: Proceedings of the 2010 IEEE Power and Energy Society general meeting, Minneapolis, 25-29 July 2010, 7 pp

[2] Verma YP, Kumar A (2012) Evaluation of emission allocation criterion for the reduction of emission in hybrid power system using PSO. In: Proceedings of the 2012 Nirma University international conference on engineering (NUiCONE'12), Ahmedabad, 6-8 December 2012, 6 pp

[3] An L, Zhao GJ (2008) Simulation of allocation pattern of carbon dioxide emission in electric sector. J Xidian Univ Soc Sci Ed 18(1):45-50 (in Chinese)
[4] He S (2012) The theory system of carbon emissions trading in power industry. $\mathrm{PhD}$ Thesis, North China Electric Power University, Beijing, pp 26-35 (in Chinese)

[5] Yang LL, Ma XC (2010) Comparison on the allocation models of carbon emission permits in electricity market. Shaanxi Electr Power 38(2):5-9 (in Chinese)

[6] He N (2013) Optimization models for the impact of carbon trading on generation permits trade in China. PhD Thesis, North China Electric Power University, Beijing, pp 41-49 (in Chinese)

[7] Jiang XC (2012) The study on carbon allowance distribution system of China. PhD Thesis, Jiangxi University of Finance and Economics, Nanchang, pp 99-112 (in Chinese)

[8] Song XD, Mo J, Xiang TY (2013) Initial allocation mechanism of carbon emission permit in electric power industry. Electr Power Autom Equip 33(1):44-49 (in Chinese)

[9] Chen Q, Jiang CW (2013) Study on carbon emission trading optimal economic efficiency of power system under different auction proportion. Water Resour Power 31(8):227-229, 246 (in Chinese)

[10] Xie CS, Dong DP, Jia XX et al (2011) Carbon emission quota allocation of electricity power sector in China: Based on emission performance. Technol Econ 30(11):57-62 (in Chinese)

[11] Wang MX, Ou BL, Wang MR et al (2011) Efficient auction mechanisms for carbon emission rights allocation. In: Proceedings of the 4th international conference on business intelligence and financial engineering (BIFE'11), Wuhan, 17-18 October 2011, pp 340-344

[12] Zhang YJ, Wang AD, Tan W (2015) The impact of China's carbon allowance allocation rules on the product prices and emission reduction behaviors of ETS-covered enterprises. Energy Policy 86:176-185

[13] Liao Z, Zhu X, Shi J (2015) Case study on initial allocation of Shanghai carbon emission trading based on shapley value. J Clean Prod 103:338-344

[14] Batlle C, Barquin J (2007) Emission rights allocation criteria. In: Proceedings of the 2007 IEEE Power Engineering Society summer meeting, Tampa, 24-28 June 2007, 3 pp

[15] Zhou X, James G, Liebman A et al (2010) Partial carbon permits allocation of potential emission trading scheme in Australian electricity market. IEEE Trans Power Syst 25(1):543-553

[16] Böhringer C, Lange A (2005) On the design of optimal grandfathering schemes for emission allowances. Eur Econ Rev 49(8):2041-2055

[17] Rosen MA (2006) An exergy-based method for allocating carbon dioxide emissions from cogeneration systems-part I: comparison with other methods. In: Proceedings of the 2006 IEEE EIC climate change technology conference, Ottawa, 10-12 May 2006, 8 pp

[18] Zheng W, Chen RD (2011). The setting of initial allocation approaches of carbon emission permits. In: Proceedings of the 4th international conference on business intelligence and financial engineering (BIFE'11), Wuhan, 17-18 October 2011, pp 668-670

[19] Keppler JH, Cruciani M (2010) Rents in the European power sector due to carbon trading. Energy Policy 38(8):4280-4290

[20] Chu JC, Zeng M, Yang LL et al (2010) Carbon emissions trading and sustainable development of power industry. In: Proceedings of the 2010 international conference on electrical and control engineering (ICECE'10), Wuhan, 25-27 June 2010, pp 3443-3446

[21] Cong RG, Wei YM (2010) Potential impact of (CET) carbon emissions trading on China's power sector: a perspective from different allowance allocation options. Energy 35(9):39213931

[22] Jaynes ET (1957) Information theory and statistical mechanics. Phys Rev 106(4):620-630 
[23] Banerjee A, Yakovenko VM (2010) Universal patterns of inequality. New J Phys 12:075032

[24] Park JW, Kim CU, Isard W (2012) Permit allocation in emissions trading using the Boltzmann distribution. Physica A 391(20):4883-4890

[25] Kong Y, Zhu DS (2013) On an intra-enterprise carbon quota distribution system. China Open J 3:36-41 (in Chinese)

Tan WANG received the B.S. degree in electrical engineering from Shanghai Jiao Tong University, Shanghai, China, in 2014. Currently, he is pursuing the M.S. degree in the School of Electronic Information and Electrical Engineering, Shanghai Jiao Tong University. His research interests include low-carbon electricity, optimization operation of power systems, and power system economics.

Xu WANG received the B.S. degree in electrical engineering from Southeast University, Nanjing, China, in 2010. Currently, he is pursuing the Ph.D. degree in the School of Electronic Information and Electrical Engineering, Shanghai Jiao Tong University, Shanghai, China. His research interests include optimization operation of power systems, electricity market, power system economics, and probabilistic power flow.

Yu GONG received the B.S. and M.S. degrees in Electric Engineering from Shanghai Jiao Tong University, Shanghai, China, in 2012 and 2015, respectively. His research interests include electricity market and low-carbon management.

Chuanwen JIANG received the M.S. and Ph.D. degrees from Huazhong University of Science and Technology, Wuhan, China, in 1996 and 2000, respectively, and completed his postdoctoral research in the School of Electronic Information and Electrical Engineering, Shanghai Jiao Tong University, Shanghai, China, in 2002. He is a Professor with the School of Electronic Information and Electrical Engineering, Shanghai Jiao Tong University. His research interests include reservoir dispatch, load forecast in power systems, and electricity market.

Fengjia XIONG received his B.S. degree of Electrical Engineering from Shanghai Jiao Tong University in 2014. He is pursuing the M.S. degree in University of California, Los Angeles, majoring in Electrical Engineering. His research interests include big data and machine learning.

Lei LI is the Senior Engineer of Shanghai Municipal Electric Power Company. His research interests include optimization operation of power systems and electricity market.

Yan ZHAO is the Senior Engineer of Shanghai Municipal Electric Power Company. Her research interests include optimization operation of power systems and electricity market. 